\title{
Hematuria Evaluation With MDCT Urography: Is a Contrast-Enhanced Phase Needed When Calculi Are Detected in the Unenhanced Phase?
}

Song JH, Beland MD, Mayo-Smith WW

Department of Diagnostic Imaging, Rhode Island Hospital-Warren Alpert Medical School of Brown University, 593 Eddy St, Providence, RI 02903

AJR Am J Roentgenol. 2011; 197: W84-9

Objective: The purpose of this study was to assess the added utility of the contrast-enhanced phase of MDCT urography (MDCTU) when urinary tract calculi are detected in the preliminary unenhanced phase.

Materials and Methods: A computer search of CT reports with the term "hematuria" yielded the records of 1209 patients who had undergone MDCTU. The reports of 286 MDCTU examinations in which urinary tract calculi were detected were identified, and two blinded abdominal radiologists reviewed the images to find a second source of hematuria. The unenhanced images were reviewed first, and the findings were compared with those on the subsequent contrast-enhanced images. The aggregate findings of the 286 examinations in which calculi were present were compared with those of the 923 examinations in which calculi were absent. The follow-up diagnosis was based on histopathologic findings, findings at urologic procedures, or the imaging diagnosis.

Results: In 119 of the 1209 patients (10\%), 127 lesions other than urinary tract calculi were identified as possible sources of hematuria. Eighty-two lesions were diagnosed in 77 patients $(6 \%)$ at follow-up evaluation. A second source of hematuria was found in 19 of the 286 examinations (7\%) with calculi compared with 58 of the 923 examinations $(6 \%)$ without calculi $(p=0.828)$, and contrast was needed to make a specific diagnosis in 16 of the 19 examinations $(84 \%)$.

Conclusion: When urinary tract calculi are identified at MDCTU, the rate of detection of other potential causes of hematuria is not different from that in MDCTU examinations without calculi. The contrast-enhanced portion of the MDCTU examination is needed even if calculi are seen because important pathologic changes are diagnosed only after the contrast-enhanced phase.

\section{Editorial Comment}

Multi-detector computed tomography (MDCT) urography has become the "gold standard" imaging technique for the investigation of patients with hematuria. MDCT urography protocol should be designed to optimize visualization of urolithiasis, the renal parenchyma and the urothelium. Although there is no consensus how to do it, most of the time, patients are imaged with the three-phase protocol: nonenhanced acquisition through the abdomen and pelvis (for detecting urolithiasis), nephrographic phase (through the abdomen) and delayed excretory phase (through the abdomen and pelvis). Using this protocol in our institution the patient effective radiation dose varies from 20-22 $\mathrm{mSv}$ (almost the double of the effective dose from an excretory urography, 10-12 mSv).

As pointed out by the authors, the American Urologic Association states that in patients with microscopic hematuria and at low risk of malignancy, no further imaging is needed if unenhanced phase of CT examination reveals urolithiasis. Since in the authors' experience, second source of hematuria had practically the same frequency in the group of patients with calculi (7\%) to those without calculi $(6 \%)$, they concluded that complete MDCT urography protocol should be performed even in these patients at low risk of malig- 
nancy. These conclusions however deserve some considerations. For example, if we assume that cystoscopy is still the reference standard for bladder cancer detection, MDCT urography would have been essential for the detection only upper tract neoplasm observed in $2 \%$ of examinations (six out 289). It is clear that this paper brought a very important contribution to the difficult task of balancing risk and benefits when performing MDCT-urography for hematuria. In our institution, for example, post-contrast phases are not obtained only in patients in who unenhanced scans reveals stone in the ureter or in the bladder. These patients and their referral physicians are instructed about the necessity of complimentary post-contrast phases only when hematuria persists after treatment or elimination of the stone.

There is no doubt that this subject is debatable and studies including patients with other risk factors are needed. Meanwhile radiologist should make efforts to reduce the radiation dose from MDCT-urography without impairing its diagnostic accuracy.

Dr. Adilson Prando

Head, Department of Radiology and

Diagnostic Imaging, Vera Cruz Hospital

Campinas, São Paulo, Brazil

E-mail: adilson.prando@gmail.com

\section{PATHOLOGY}

Significance of Prostate Adenocarcinoma Perineural Invasion on Biopsy in Patients Who are Otherwise Candidates for Active Surveillance

Al-Hussain T, Carter HB, Epstein JI

Department of Pathology, The Johns Hopkins Hospital, Baltimore, Maryland

J Urol. 2011; 186: 470-3

Purpose: Perineural invasion on biopsy is associated with extraprostatic extension at radical prostatectomy. To our knowledge the significance of perineural invasion on biopsy in patients who otherwise meet the criteria for active surveillance has not been studied.

Materials and Methods: The biopsy criteria for active surveillance were Gleason score 6 or less, 2 or fewer positive cores and $50 \%$ or less involvement any positive core. All cases had at least 12 biopsy cores. A total of 313 cases met the biopsy criteria for active surveillance, and elected to undergo immediate radical prostatectomy at our institution between 1992 and 2008. These cases included 51 with perineural invasion and 262 without perineural invasion.

Results: There was no significant difference in patient age and mean serum prostate specific antigen at diagnosis in cases with and those without perineural invasion. Cases with perineural invasion on biopsy had a higher maximum percentage of cancer on biopsy $(18.6 \%)$ vs those without perineural invasion $(15.0 \%, \mathrm{p}=$ 0.02). Cases with perineural invasion also had slightly more with 2 positive cores compared to cases without perineural invasion $(56.9 \%$ and $39.7 \%$, respectively, $\mathrm{p}=0.02)$. Despite a greater extent of cancer on biopsy, cases with and those without perineural invasion on biopsy showed no significant difference in surgical margin involvement ( $6 \%$ vs $7.3 \%$, respectively) or organ confined disease ( $84.3 \%$ vs $91.6 \%$, respectively). Conclusions: Cases that meet biopsy criteria for active surveillance yet have perineural invasion showed no significant difference from those without perineural invasion in terms of adverse findings at radical 